\title{
Adaptive driving beam system with MEMS optical scanner for reconfigurable vehicle headlight
}

\author{
Tomotaka Asari, ${ }^{a}$ Mamoru Miyachi, ${ }^{a}$ Yutaro Oda, ${ }^{\text {a }}$ Takaaki Koyama, ${ }^{a}$ \\ Hiroaki Kurosu, ${ }^{a}$ Makoto Sakurai, ${ }^{a}$ Masanao Tani, ${ }^{a}$ Yoshiaki Yasuda, ${ }^{a}$ \\ and Hiroshi Toshiyoshi $\odot^{b, *}$

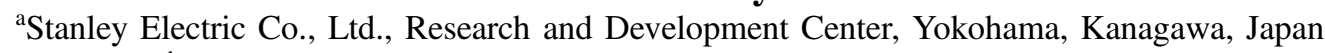 \\ ${ }^{b}$ The University of Tokyo, Institute of Industrial Science, Tokyo, Japan
}

\begin{abstract}
An adaptive driving beam (ADB) for vehicle headlights has been developed using a microelectromechanical systems optical scanner. A piezoelectric scanner is constructed using thin-film lead-zirconate-titanate oxide $\left(\mathrm{PbZrTiO}_{3}, \mathrm{PZT}\right)$ on a bonded silicon-on-insulator (SOI) wafer, respectively, processed by ion-milling and deep reactive ion etching. The PZT layer is laminated in metal films on the SOI layer to form a piezoelectric unimorph actuator, which are then arranged as a pair of twisting suspensions to drive the scanner at resonance. The same piezoelectric actuators are also arranged into another form of meandering suspensions to generate a large deflection angle. By the combination of these two mechanisms, two-dimensional optical scanner is constructed in a single chip. The scanner is used to draw a Lissajous pattern of a blue laser light on a phosphor material to create a structured light source that is projected forward for illumination. The lighting patterns and positions are electronically controlled and reconfigured depending upon the location of leading/oncoming vehicles, pedestrians, road signs, and the cruising speed of the vehicle. The paper discusses on the design of the piezoelectrically driven optical scanner along with the optomechanical performances. We also report on the road-test result of the developed on-vehicle ADB module. (C) The Authors. Published by SPIE under a Creative Commons Attribution 4.0 Unported License. Distribution or reproduction of this work in whole or in part requires full attribution of the original publication, including its DOI. [DOI: 10.1117/1.JOM.1.1 .014501]
\end{abstract}

Keywords: microelectromechanical systems; scanner; piezoelectric; lead-zirconate-titanate; headlight; vehicle.

Paper 20011 received Sep. 22, 2020; accepted for publication Jan. 4, 2021; published online Jan. 26, 2021.

\section{Introduction}

Adaptive driving beam (ADB) is an advanced drive-assist technology for an automobile headlight that provides drivers with visibility of high contrast and illuminance at night without causing temporary blindness to other drivers in a projected light's glare. ${ }^{1,2}$ Different from the classic headlight with manually switchable low- and high-beam modules, the ADB system throws reconfigurable light depending upon the cruising speed and the traffic environments such as presence of oncoming or leading vehicles, pedestrians, and traffic signs that are automatically recognized by the on-board computer vision system. Due to the fact that mortality rate of accidents at night is two to three times higher than that in daytime, ${ }^{3}$ enhancement of visibility at night has been deemed a crucial issue for a long time. Supported by the recent development of dashboard cameras for visual recognition and solid-state light sources in the headlamp module, a microcomputer-controlled ADB system has become technologically feasible as a part of onvehicle electronics.

An array of light-emitting diodes, ${ }^{4-6}$ for instance, is used in the commercialized ADB optics that changes the projected light patterns in a digital manner. A sliding shade can also be used in front of a light bulb to locally block the light. Due to the few degrees of controllability freedom in such systems, nevertheless, the patterns of projected light are limited in a zonal shape as schematically shown in Fig. 1(a), sometimes resulting in an excessive black-out area $(H \times W)$ that

*Address all correspondence to Hiroshi Toshiyoshi, hiro@iis.u-tokyo.ac.jp 


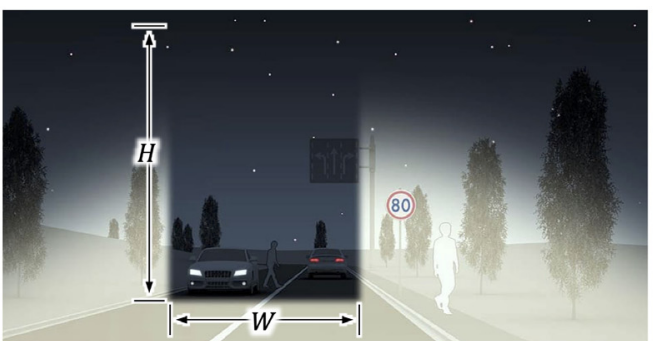

(a)

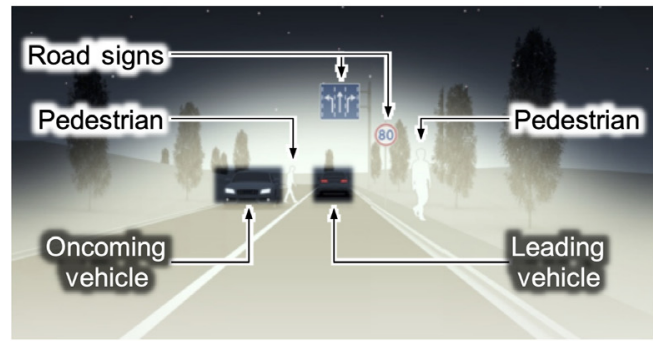

(b)

Fig. 1 Effects of ADB for enhanced visibility. (a) Conventional zonal shielding that excessively masks a road sign and a pedestrian. (b) Spot shielding for oncoming and leading vehicles.

rather obscures the visibility of the road signs and nearby pedestrians. A two-dimensional array of liquid crystal pixels ${ }^{7}$ or digital micro mirrors ${ }^{8,9}$ is thus employed as a spatial light modulator (SLM) in recent ADB system of high degree of programming flexibility. Creating of more complicated illumination patterns is possible with the SLM, as shown in Fig. 1(b), owing to the optics similar to the image projection display. Despite the extra capabilities such as showing information on the road, nevertheless, the SLM-based optics usually becomes costly. Due to the SLM mechanism that is based on light blocking, the light power that is not projected forward is consumed as heat loss in the headlight module. Therefore, the coefficient of light power utilization drops with increasing the black-out area, resulting in the requirement of more margin in the thermal design of the ADB system.

As an alternative solution for ADB, we have developed a microelectromechanical system (MEMS) two-dimensional optical scanner to produce various illumination patterns on a phosphor luminescent material, which are projected forward through the imaging lenses. A thin-film lead-zirconate-titanate oxide $\left(\mathrm{PbZrTiO}_{3}, \mathrm{PZT}\right)$ film is used to piezoelectrically excite the mechanical oscillation of the scanning mirror to draw Lissajous patterns in synchronization with a laser diode (LD). In this paper, we discuss the mechano-electric structures of the optical scanner along with the fundamental oscillation performance that has been tuned for vehiclemounted applications. A test module of ADB headlight is assembled with the scanner, and field-tested to demonstrate the cruise-speed dependent illumination and the pedestrian recognition by the patterned lighting. This paper includes the extended results based on the technical reports presented at IEEE OMN $2019^{10}$ and ISAL 2019. ${ }^{11}$

\section{ADB Optics}

Figure 2(a) shows the ADB system architecture that has been developed in this work. A collimated beam of an LD (wavelength $450 \mathrm{~nm}$, power $3.5 \mathrm{~W} \max$ ) is spatially steered by a

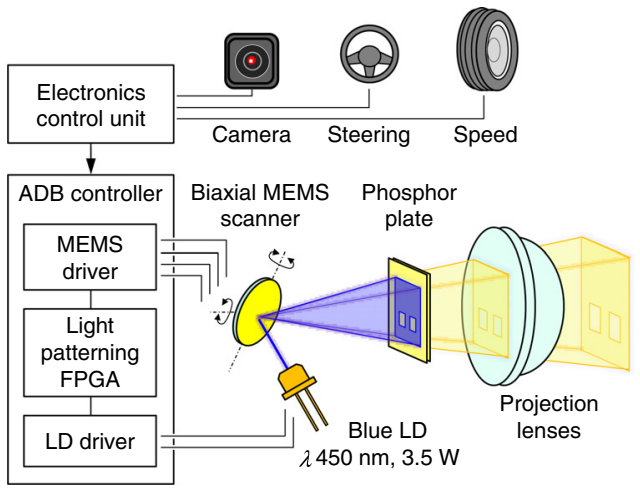

(a)

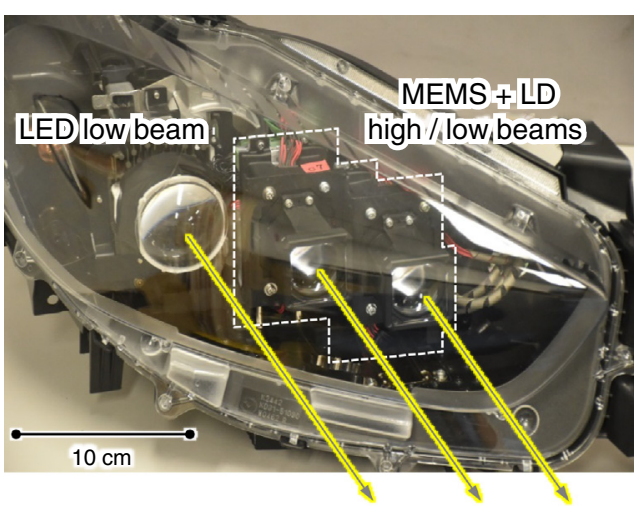

(b)

Fig. 2 Optics for ADB. (a) System block diagram and (b) implementation in the head lamp block. 
two-dimensional MEMS optical scanner to form a structured light sheet on the phosphor plate, where the blue light is converted into a white-light pattern of high brightness, which is subsequently threw forward through the projection lenses. The digital ADB controller reads in the state variables such as image recognized by the on-board camera, the steering wheel angle, and the cruising speed of the vehicle, and commands the operation voltages of the scanner and the injection current of the LD. The intensity of the LD is modulated in synchronization with the MEMS scanner to create Lissajous bitmap images of a Quarter Video Graphics Array resolution or equivalent.

Owing to the fast response of the LD, the projected light can contain fine black-out spots at arbitrary positions as commanded by the field programmable gate array (FPGA) logic. Given a constant power of the $\mathrm{LD}$, the luminance of projection is determined by the scanned area on the phosphor plate; the spot brightness increases when the excitation light is focused in a small spot, while the brightness decreases when the excitation is diluted by painting a large area on the phosphor plate. The total power of the blue light is thus converted into the projection light at a high usage efficiency, which is not achievable by the optics based on the digital mirror or liquid crystal pixels because the light blocked by such an SLM is eventually consumed as heat in the ADB system.

Figure 2(b) shows the photograph of an assembled headlight block to be mounted to the right-hand side of a vehicle; two modules out of three, surrounded by the dashed lines, are the MEMS-ADBs that cover the high- and low-areas of illumination in combination. Final product may use a single MEMS module but two modules are used in this R\&D phase for the variety of trials. Owing to the relatively large scan angle of the MEMS, each optics is packaged in a small volume of $7 \times 7 \times 10 \mathrm{~cm}^{3}$ or less. Thermal management of the device should be carefully designed because the on-vehicle electronics are supposed to operate in a wide temperature range from $-40^{\circ} \mathrm{C}$ to $+125^{\circ} \mathrm{C}$, which is more stringent than that for most consumer electronics, from $-40^{\circ} \mathrm{C}$ to $+85^{\circ} \mathrm{C}$.

\section{MEMS PZT Optical Scanner}

\subsection{Device Design}

Among various electromechanical actuation principles including electrostatic, ${ }^{12}$ electromagnetic, ${ }^{13}$ and electrothermal, ${ }^{14}$ we have chosen the piezoelectric actuation mechanism for the MEMS optical scanner for its large areal density of force achievable in a relatively low voltage ${ }^{15}$ In addition, piezoelectric actuation mechanism does not require a fine air gap that is inevitably needed in the electrostatic actuation, which may become vulnerable to thermal expansion at a high temperature.

A scanning electron microscope (SEM) image of the scanner is shown in Fig. 3(a). The entire chip is made of a bonded silicon-on-insulator (SOI) wafer with an active silicon layer of $50 \mu \mathrm{m}$ in thickness and a thin film piezoelectric layer formed on it. The disk in the middle is a mirror of

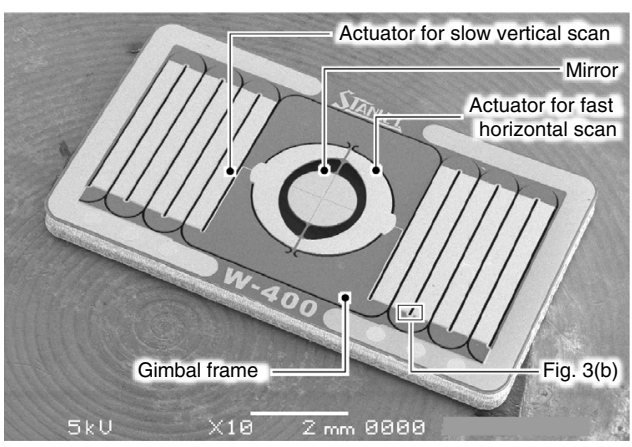

(a)

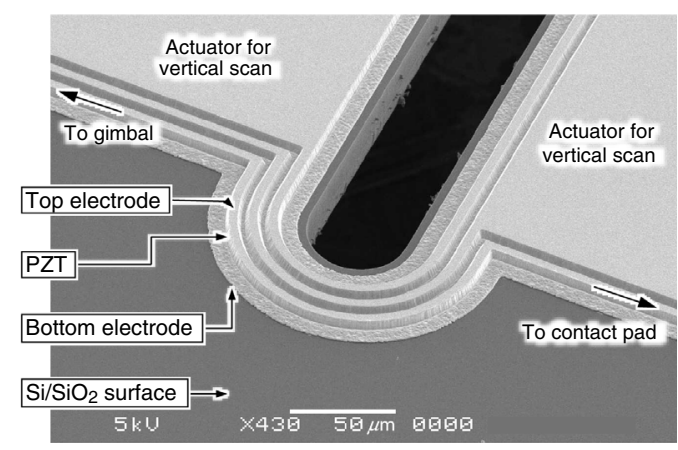

(b)

Fig. 3 SEM images of MEMS optical scanner. (a) Entire chip view and (b) close-up image of the bending corner of meandering suspensions. 


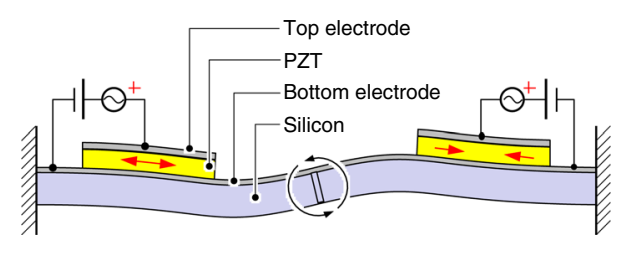

(a)

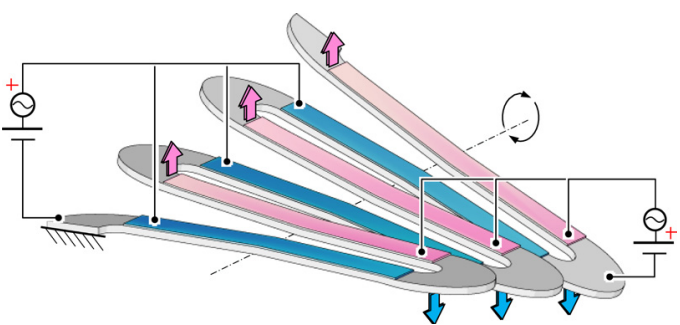

(b)

Fig. 4 Application of piezoelectric unimorph actuation. (a) Twisting torsion bar using a pair of bending beams and (b) serial connection of bending beams to accumulate the angular deflection.

$1.5 \mathrm{~mm}$ in diameter, which is supported by a pair of torsion beams of $50 \mu \mathrm{m}$ in width, $200 \mu \mathrm{m}$ in length, and $50 \mu \mathrm{m}$ in thickness. The light-gray areas in the SEM correspond to the structures with metallization for actuators, electrical interconnections, and mirror's reflector. In particular, the structures in a crescent shape are the pair of PZT unimorph actuators to excite the resonance of the mirror disk about the rotation axis of the torsion bars. The square block that contains the mirror and the crescent actuators is a gimbal frame, which is supported by a pair of meandering suspensions of $300 \mu \mathrm{m}$ in width and $4 \mathrm{~mm}$ in length. The combination of the tilt motion of the gimbal and the out-of-plane rotation of the mirror make up two-dimensional scan for beam steering. A close-up SEM image of the bending corner of the meandering suspensions is shown in Fig. 3(b), where three co-centric arc patterns are seen; these are the electrical interconnection lines of thin-film metals, under which a PZT layer is embedded.

The actuation principle is shown in Fig. 4(a). The silicon beam coated with a metal layer serves as a bottom electrode for the piezoelectric unimorph actuator. The top electrodes are DCbiased in the same polarity, while differential AC voltages of opposite polarities are superposed to it. Therefore, one of the PZT actuators produces longitudinal compressive stress in the upper skin of the beam, while the other one gives tensile stress, thereby bending the beam in an S-shape to twist the torsion bar attached in the middle; this twist motion is transferred to the mirror disk to excite mechanical resonance for the fast horizontal scan.

The slow scan in the orthogonal direction is produced by the cascaded PZT cantilevers as shown in Fig. 4(b). The cantilevers are mechanically connected in one piece to form a meandering suspension, and the individual electrodes are electrically connected in every other position, thereby forming two groups of electrically independent actuators arranged in an alternating format. By applying AC voltages of opposite polarities superposed on a common DC-bias voltage, the cantilevers in one group deflect downward while those in the other group go upward. The angular displacement of each beam is thus accumulated in the cascaded structure, finally yielding a large deflection angle at the tip. The gimbal frame of the scanner is actuated by this mechanism around the rotation axis orthogonal to the fast horizontal scan.

\subsection{Fabrication Process}

We have designed the fabrication process steps as straightforward as possible as shown in Fig. $5 ;{ }^{15}$ all the thin film deposition processes are performed in the early part of the steps, while the latter half is mostly for patterning the films. Owing to this process scheme, silicon wafers with a PZT layer could be made-ahead in quantity to lower the production cost.

In step (1), the process begins with an SOI wafer with a 50- $\mu$ m-thick silicon layer, a $1-\mu \mathrm{m}$ thick buried oxide (BOX) layer, and a 525- $\mu \mathrm{m}$-thick handle silicon layer. Silicon oxide of $\sim 1 \mu \mathrm{m}$ in thickness is formed on the surface by the wet oxidation. In step (2), a 150-nm-thick platinum layer is formed on a 50-nm-thick titanium layer as an adhesion promotor; these layers work as the bottom electrode for piezoelectric actuation. Subsequently, a 3- $\mu$ m-thick PZT is deposited by the arc discharge reactive ion plating process, ${ }^{16}$ which is a sputtering process with an auxiliary arc-discharge source to electrochemically activate the sputtered species for better quality of piezoelectric properties. Step (3) is the patterning of the top electrode, the PZT, and the bottom electrode all done by the ion milling through the photoresist masks. Another photomask is used 

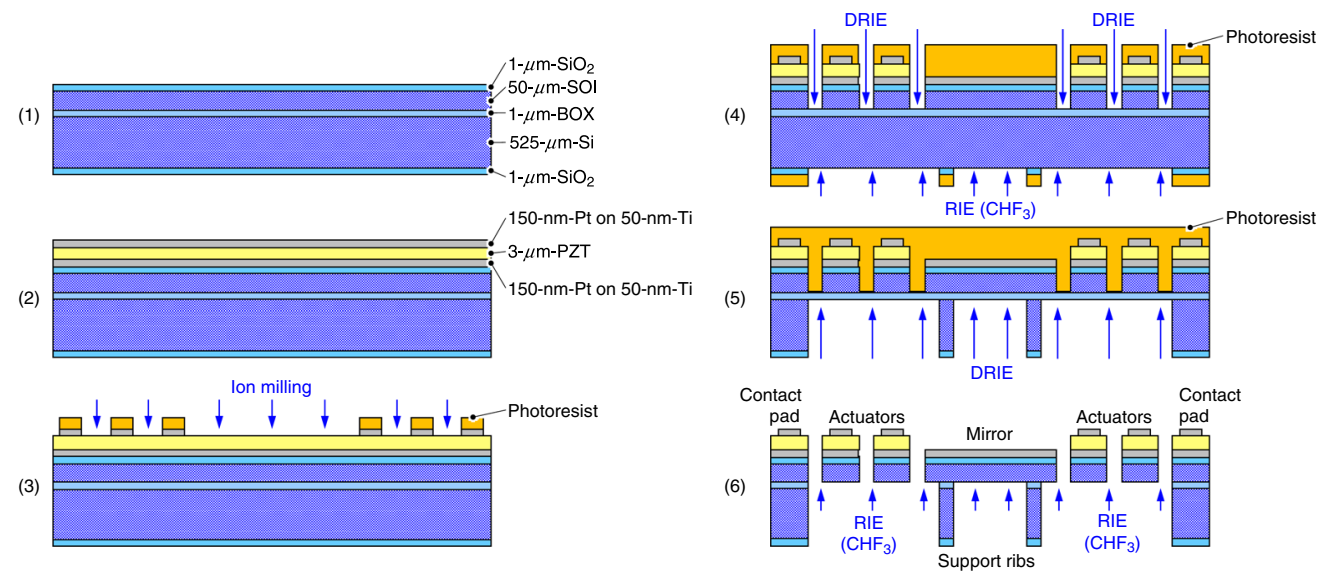

Fig. 5 Fabrication process steps of piezoelectric MEMS optical scanner.

in step (4) to further extend the top side etching into the SOI layer by the deep reactive ion etching (DRIE) until stopped by the BOX layer. By this step, the fundamental structures of the mirror, the torsion bars, and piezoelectric unimorph actuators have been formed. Protecting these fine structures under a passivation photoresist layer in step (5), the silicon oxide on the reverse side is patterned by reactive ion etching (RIE) using $\mathrm{CHF}_{3}$ to form an etching mask for the DRIE process of the silicon substrate. Finally, in step (6), the BOX layer is selectively etched from behind by the RIE using a $\mathrm{CHF}_{3}$ gas, and the passivation photoresist is removed by the oxygen plasma to finish the release of movable structures in a dry phase. The patterned silicon structure remaining on the backside of the mirror is used to keep the mirror surface as flat as possible. The shape of the backside support is determined using the structure optimization tool that takes the mechanical strength and the moment of inertia into the consideration of mirror's flatness and resonant frequency. ${ }^{17}$

\subsection{Device Characterization}

Scanner's piezoelectric actuation performance was tested in room temperature under the standard $1 \mathrm{~atm}$ pressure. Figure 6(a) shows the mechanical half angle of the mirror about the fast axis excited at the fundamental resonance of $21.29 \mathrm{kHz}$ by the unipolar differential voltages applied to the pair of crescent-shape actuators. The maximal half-mechanical angle was 14 deg when excited by $7.5 V_{d c} \pm 7.5 V_{a c}$, meaning that the optical full-width angle would be as large as $56 \mathrm{deg}$ for the surface-normal incident; the fast axis with a relatively wide scan angle was therefore used for the horizontal scan for the headlight projection.

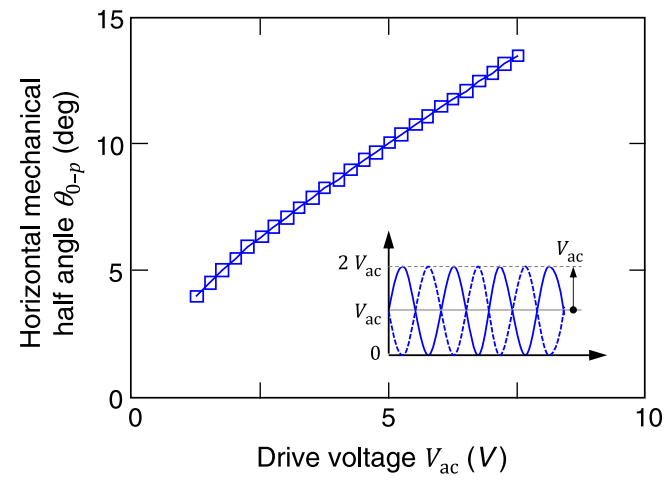

(a)

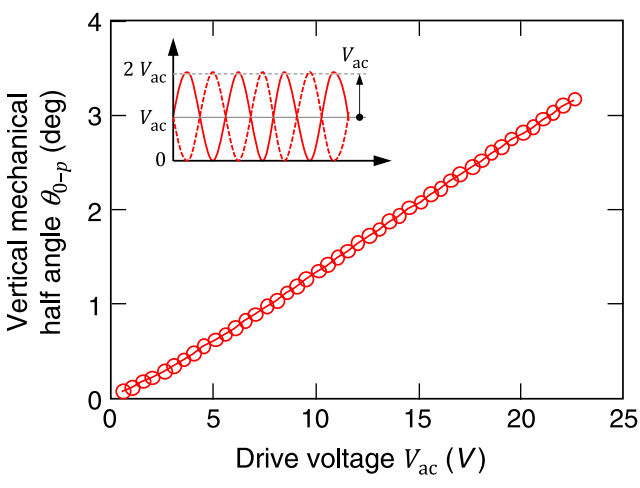

(b)

Fig. 6 Scanner's mechanical deflection angles as a function of applied voltage. (a) Horizontal fast axis around the torsion beam and (b) vertical slow axis at the tip of meandering beams. 
On the other hand, the meandering suspensions were used for the vertical axis operated at a relatively low frequency of $60 \mathrm{~Hz}$. The deflection angle was controlled with good linearity as shown in Fig. 6(b), when excited by the similar unipolar differential voltages. The maximal halfmechanical angle was as large as $3 \mathrm{deg}$ when actuated by $23 \mathrm{~V}$ even under a DC-voltage operation.

The frequency spectrum of the fast axis angle was of a quadratic harmonic oscillator as shown in Fig. 7(a). Typical resonant frequency was found at $21.3 \mathrm{kHz}$ with a full-width-athalf-maximum of around $43 \mathrm{~Hz}$, corresponding to a quality factor of 500. The scanner's oscillation angles were monitored by the built-in piezoelectric sensors reported elsewhere ${ }^{17}$ to compose a feed-back control system.

Optical scanner for the ADB must not be disturbed by the external vibrations that are inherent to the vehicle operation including the running engines and the rotating tires. It is empirically known that vehicle noises are found in a frequency range lower than $1 \mathrm{kHz}$, and therefore the resonant modes of the scanner were carefully designed not to react to such low-frequency excitation. The scanner's frequency response to the external vibration was experimentally observed on a shaker, as shown in Fig. 7(b); the distinguishable peaks were of the higher mode resonances of the meandering suspensions. Judging from the flat response in the frequency lower than $1 \mathrm{kHz}$, the scanner was expected to be robust against vehicle's mechanical disturbances.

As shown in Fig. 8(a), piezoelectric $d_{31}$ constant of the developed PZT film was confirmed to be stable within 220 to $230 \mathrm{pm} / \mathrm{V}$ in a wide temperature range from $20^{\circ} \mathrm{C}$ to $140^{\circ} \mathrm{C}$. Long-term test on the piezoelectric $d_{31}$ constant exhibited only $-2 \%$ aging degradation after $8000 \mathrm{~h}$ at $125^{\circ}$ C. PZT materials usually have a high phase transition temperature of around $340^{\circ} \mathrm{C}$, implying a large margin to the highest operation temperature. In addition, temperature dependence of the

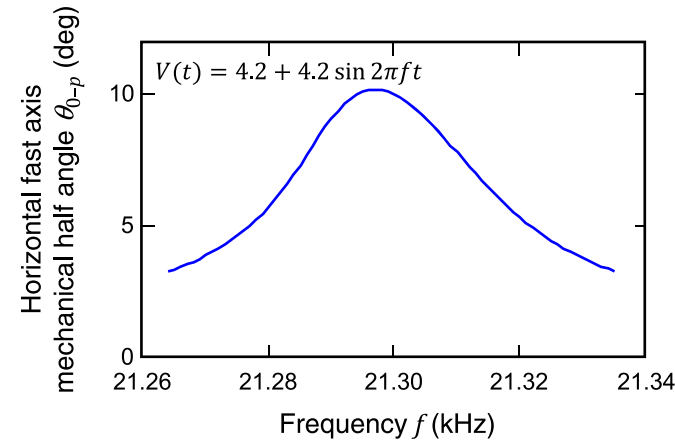

(a)

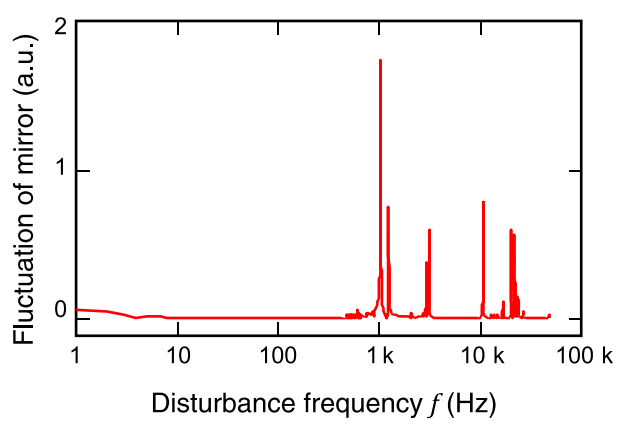

(b)

Fig. 7 Scanner's frequency response. (a) Horizontal fast-axis response to the built-in piezoelectric actuation. (b) Mirror oscillation when excited by external vibrations.

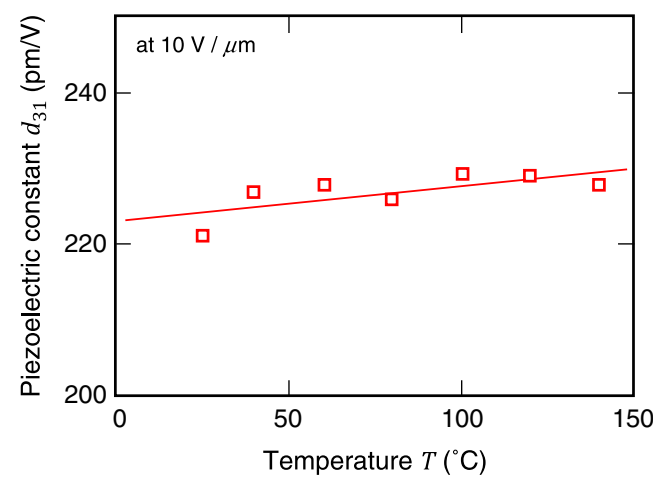

(a)

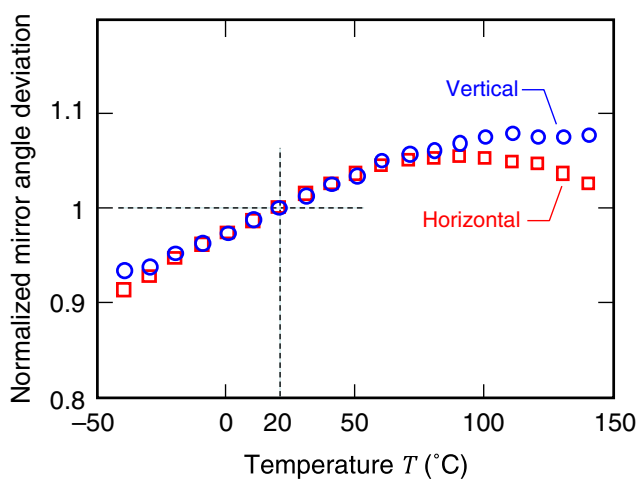

(b)

Fig. 8 Temperature dependence. (a) Piezoelectric $d_{31}$ constant and (b) mirror's deflection angles. 
deflection angle was characterized between $-40^{\circ} \mathrm{C}$ and $+140^{\circ} \mathrm{C}$ as shown in Fig. 8(b), which was found to be reproducible for $5000 \mathrm{~h}$. The ADB control system was designed to compensate the effect of temperature by reading the temperature inside the head lamp module. Owing to the mechanical reinforcement structure on the backside, the peak-to-valley deformation of the mirror at $180^{\circ} \mathrm{C}$ was found to be no greater than $30 \mathrm{~nm}$, which was sufficiently small for the operation as a scanner for ADB.

\section{Demonstration}

The ADB module assembled with the developed PZT scanner was mounted on a vehicle, and its performance was road-tested at night. Figure 9 compares the lighting patterns projected to an oncoming vehicle (a) with and (b) without using the image recognition function of the ADB system. In both cases, the lighting was adjusted not to throw a glare light to the driver on the oncoming vehicle, whose position was automatically tracked by the visual recognition system linked to the on-board camera. By appropriately adjusting the black-out spot for the oncoming vehicle at a high angular resolution better than $0.1 \mathrm{deg}$, a pedestrian standing by the car was clearly profiled in the light as shown in Fig. 9(a). When the image recognition was turned off, on the other hand, the pedestrian became incognizable in the black-out area that had been excessively set large for a low-beam configuration as shown in Fig. 9(b). The ADB with a MEMS scanner was thus found to tune the black-out area in illumination at a high resolution to provide the driver with better visibility.

Another use of ADB is to control the lighting area depending upon the cruising speed. At a low speed, drivers usually pay attention to the nearby objects, particularly when turning the corners, where a rather broadside illumination is required. When at a fast speed, on the other hand, they opt to look into the far distance, and a spot illumination of high intensity is favored. We programmed the ADB system so that the drivers would receive at least 3-lux-light backscattered from an object ahead, and controlled the lateral fan-out angle as well as the depression angle of projection. Figure 10 compares the illumination distance adapted to different cruising speeds as $190 \mathrm{~m}$ ahead at $40 \mathrm{~km} / \mathrm{h}$ [Fig. 10(a)], $250 \mathrm{~m}$ ahead at $60 \mathrm{~km} / \mathrm{h}$ [Fig. 10(b)], and $338 \mathrm{~m}$ ahead at $90 \mathrm{~km} / \mathrm{m}$ [Fig. 10(c)]; the illumination area of high intensity could be known by the shining reflectors on the guardrail. In the ADB optics for a low speed, the MEMS scanner was controlled to scan a relatively wide lateral area on the phosphor material to illuminate a large area. At high speed, the scan angle was confined within a small area to obtain high-intensity luminescence to throw a bright light over the distance.

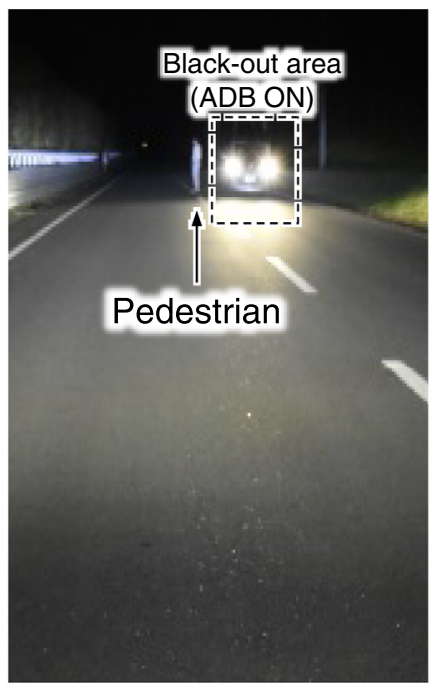

(a)

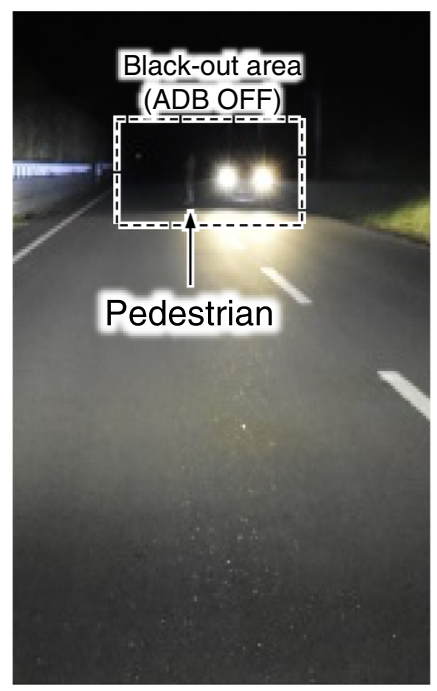

(b)

Fig. 9 Road test of ADB projection. (a) ADB ON. Pedestrian profiled by the light. (b) ADB OFF. Incognizable pedestrian in the black-out area. 


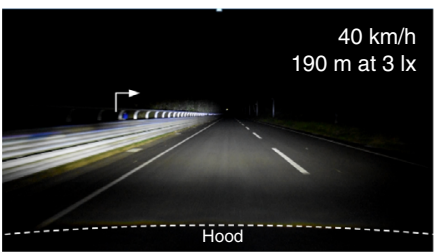

(a)

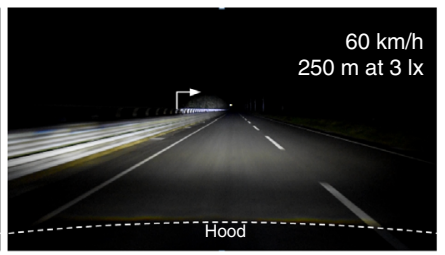

(b)

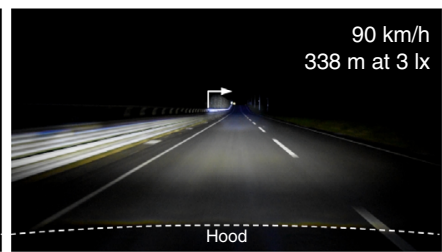

(c)

Fig. 10 Road test of cruise-speed dependent ADB. Light projection at (a) $190 \mathrm{~m}$ ahead at $40 \mathrm{~km} / \mathrm{h}$; (b) $250 \mathrm{~m}$ at $60 \mathrm{~km} / \mathrm{h}$; and (c) $338 \mathrm{~m}$ at $90 \mathrm{~km} / \mathrm{h}$ for at least 3-lux visibility.

\section{Conclusion}

In this paper, we discussed a new use of the piezoelectrically driven MEMS optical scanner in an ADB system for vehicle, where the scanner was employed to create two-dimensional phosphor patterns that were projected forward for adaptive lighting. With the development of the automatic visual recognition technology, the ADB has become a part of the autonomous driving system to provide the driver with the best illumination patterns by automatically recognizing the leading/ oncoming vehicles, pedestrians, and road signs. For demonstration, we performed adaptive lighting to visualize a pedestrian standing by the oncoming vehicle without projecting a glare light to the other driver. We also demonstrated the reconfigurable lighting depending upon the cruising speed of the vehicle. Headlight block of modern vehicle has thus become advanced by including the adaptive beam using the MEMS optical scanners. The system could be further extended to include the function of LiDAR (Light Detection And Ranging) for range-finding and the function of inter-vehicle optical communication links that could be used in the self-driving technology of advanced intelligent traffic systems (ITS).

\section{Acknowledgments}

A part of this research was supported by the Japan Society for the Promotion of Science (JSPS) through the "Funding Program for Next Generation World-Leading Researchers (NEXT Program)," initiated by the Council for Science and Technology Policy (CSTP), Grant No. GR024. The authors declare no conflict of interest.

\section{References}

1. J. Bhardway and B. Spinger, "What will mainstream advanced driving beam look like by 2025?” Proc. SPIE 10940, 1094000 (2019).

2. I. J. Reagan and M. L. Brumbelow, "Perceived discomfort glare from an adaptive driving beam headlight system compared with three low beam lighting configurations," Procedia Manuf. 3, 3214-3221 (2015).

3. U.S. National Highway Traffic Safety Administration (NHTSA), "Traffic Safety Facts Annual Report Tables 2018," https://cdan.nhtsa.gov/tsftables/tsfar.htm (accessed 9 September 2020).

4. "Adaptive driving beam headlamp for vehicle," United States Patent US9,873,372 B2 (2018).

5. H.-Y. Kim et al., "Optimization of InGaN-based LED package structure for automotive adaptive driving beam headlamp," ECS J. Solid State Sci. Technol. 9, 055017 (2020).

6. Koito Manufacturing Co., Ltd., "The newest lighting system," https://www.koito.co.jp/ english/technology/koito/system.html (accessed 9 September 2020).

7. C. J. Reinert-Weiss et al., "Development of active matrix LCD for use in high-resolution adaptive headlights," J. SID 25(2), 90-97 (2017).

8. P. F. Aclantarilla et al., "Night time vehicle detection for driving assistance lightbeam controller," in IEEE Intell. Veh. Symp., Eindhoven University of Technology, Eindhoven, The Netherlands, pp. 291-296 (2008). 
9. "Pixelated projection for automotive headlamp," United States Patent US10,066,799 B2 (2018).

10. T. Asari et al., "A bi-axial piezoelectric MEMS scanning mirror for automobile lighting system," in Proc. Int. Conf. Opt. MEMS and Nanophotonics, KAIST, Daejeon, South Korea, pp. 224-225 (2019).

11. M. Miyachi et al., "Development of laser scanning headlamps using MEMS mirror device," in Proc. 13th Int. Symp. Automotive Lighting, Darmstadt, pp. 515-524 (2019).

12. S. Hsu et al., "Fabrication and characterization of a dynamically flat high resolution microscanner," J. Opt. A Pure Appl. Opt. 10, 044005 (2008).

13. C.-H. Ji et al., "Electromagnetic two-dimensional scanner using radial magnetic field," J. Microelectromech. Syst. 16(4), 989-996 (2007).

14. W. Liao et al., "A tip-tilt-piston micromirror with symmetrical lateral-shift-free piezoelectric actuators," IEEE Sens. J. 13(8), 2873-2881 (2013).

15. M. Tani et al., "A two-axis piezoelectric tilting micromirror with a newly developed PZTmeandering actuator," in Proc. 20th IEEE Int. Conf. Micro Electro Mech. Syst., Kobe Portopia Hall and Kobe Portopia Hotel, Japan (2007).

16. Y. Yasuda et al., "Preparation of lead zirconate titanate thick films by arc-discharged reactive ion-plating method," Jpn. J. Appl. Phys. 40(Pt. 1, No. 9B), 5518-5522 (2001).

17. K. Ikegami et al., "A biaxial PZT optical scanner for pico-projector applications," Proc. SPIE 9375, 93750M (2015).

Tomotaka Asari received BS and MS degrees in physics from Yokohama National University in 2008 and 2010, respectively. He joined Seiko Epson Corporation in 2010 and worked on the development of quartz gyroscopes. Since 2016, he has been engaged in the development of MEMS scanners at Stanley Electric Co., Ltd.

Hiroshi Toshiyoshi received his $\mathrm{ME}$ and $\mathrm{PhD}$ degrees in electrical engineering from the University of Tokyo, Tokyo, Japan, in 1993 and 1996, respectively. He joined the Institute of Industrial Science, the University of Tokyo, in 1996. From 1999 to 2001, he was a visiting assistant professor at the University of California, Los Angeles, California, USA. Since 2009, he has been a professor. His research interests include optical MEMS, power MEMS, and CMOSMEMS.

Biographies of the other authors are not available. 